\title{
Customised micro-electrode array (MEA) test setup featuring a sili- cone-casted overlay with two chambers for separated cell seedings
}

\author{
Robert $\mathrm{Mau}^{1+}$, Sophie Kussauer ${ }^{2,3+}$, Uta Matzmohr ${ }^{1}$, Robert David ${ }^{2,3 *}$ and Hermann Seitz ${ }^{1,3 *}$ \\ ${ }^{1}$ Microfluidics, University of Rostock, Rostock, Germany \\ ${ }^{2}$ Department Cardiac Surgery, Rostock University Medical Center, Rostock, Germany \\ ${ }^{3}$ Department of Life, Light and Matter, University of Rostock, Rostock, Germany
}

${ }^{\star}$ Corresponding authors: Prof. Dr.-Ing. Hermann Seitz, hermann.seitz@uni-rostock.de, Prof. Dr. rer. nat. Robert David robert.david@med.uni-rostock.de

${ }^{+}$These authors contributed equally to this manuscript

\section{Introduction}

Micro-electrode array (MEA) systems are non-invasive platforms for the investigation of electrophysiological properties of cell layers, such as spontaneously active cardiomyocytes. An MEA chip is composed of two-dimensional grids of dot-like electrodes embedded into glass. Here we present a test setup featuring a customised two-chamber silicone overlay to be placed on a MEA with two fields of electrodes. Silicone is an excellent biocompatible material, suitable for long term cell contact with user-friendly processability. The overlay enables the seeding of two separated cell subtypes on the MEA for synchronised drug testing applications while giving the possibility to analyse intersubtype- specific cellular interactions.

\section{Methods}

A customised overlay with two chambers was realised via silicone casting. First, a model of the overlay $(10 \mathrm{~mm} \times 10 \mathrm{~mm})$ was designed via CAD and 3D-printed via high-resolution digital light processing (DLP, VIDA device, Envisiontec, Germany). E-Model light (Envisiontec, Germany) was used for 3D printing, which is a photopolymer material for stiff, water-resistant and high-detailed modelling needs. Second, the 3D-printed model was used for the casting of shrink-free molds, made of two-component silicone rubber, vulcanised at room temperature with a cure time of $24 \mathrm{~h}$. Next, the molds were used for casting the final overlay with the biocompatible silicone. After casting, it was vulcanised for 48 hours at room temperature. Finally, a cover for optimised attachment of the overlay on the MEA was 3D printed via DLP using Class-IIa biocompatible E-Shell 600 material (Envisiontec, Germany).

\section{Results}

Reasoned by the given design of the two field MEA chip (multi-channel systems), a small wall thickness of the silicone overlay of $300 \mu \mathrm{m}$ is required. Nevertheless, the customised two-chamber silicone overlay was realised successfully. Precise attachment of the silicone overlays on glass MEA can be realised manually via utilising a light microscope. The cover provides a stable attachment and a water-proof sealing of the two chambers to ensure that there is no mixture of cell media between the chambers. Customised openings in the cover enable media change as well as oxygen supply.

\section{Conclusion}

An MEA test setup utilising a customised silicone overlay $(10 \times 10 \mathrm{~mm}$, wall thickness of $300 \mu \mathrm{m})$ with two chambers for separated cell seedings was successfully developed. Further work will focus on a coating of the silicone overlay to prevent adhesion of hydrophobic substances prior to drug testing in cardiac cells. 


\title{
Utilizing direct-initiation of thiols for photoinitiator-free stereolitho- graphic 3D printing of mechanically stable scaffolds
}

\author{
Klaus Kreuels, Chair for Laser Technology LLT, RWTH Aachen University, Aachen, Germany, \\ klaus.kreuels@1lt.rwth-aachen.de \\ David Bosma, Chair for Laser Technology LLT, RWTH Aachen University, Aachen, Germany \\ Nadine Nottrodt, Fraunhofer Institute for Laser Technology ILT, Aachen, Germany, nadine.nottrodt@ilt.fraunhofer.de \\ Arnold Gillner, Chair for Laser Technology LLT, RWTH Aachen University and Fraunhofer Institute for Laser \\ Technology ILT, Aachen, Germany, arnold.gillner@ilt.fraunhofer.de
}

\section{Introduction}

Additive manufacturing enables the production of scaffolds as complex structures to mimic biomechanically complex tissues. Hydrogel 3D printing to mimic the direct cell environment is widely known in the field of biofabrication and photoinitiator-free 3D printing for higher biocompatibility is also demonstrated. However, complex structures for the fabrication of larger tissues that meet the biomechanical requirements are still challenging. In our work we present the photoinitiator-free stereolithographic production of thiol-ene polymer scaffolds ranging in size from tens of microns in the microarchitecture to millimeters on the large scale. Furthermore, these scaffold structures exhibit dimensional stability and load bearing properties as probed by compression testing.

\section{Methods}

The photo resin used is a biocompatible material system composed of trimethylolpropane tris(3-mercaptopropionate) (TMPMP) and poly(ethylene glycol) divinyl ether (PEG-DV) in stoichiometric equivalent amounts with respect to the functional thiol and ene groups, respectively. Thiol-ene polymer scaffolds were manufactured using a custom-made stereolithographic setup with a continuous wave, frequency quadrupled Nd:YAG laser source at a wavelength of $\lambda=266 \mathrm{~nm}$. Obtained polymer structures were analyzed by laser scanning microscopy to determine the working parameters and the photopolymerization reaction was investigated by FTIR spectroscopy. Thoroughly cleaned scaffolds were characterized mechanically by compression testing.

\section{Results}

FTIR spectroscopy confirms the formation of a thiol-ene polymer upon a photopolymerization using laser radiation at $\lambda=266 \mathrm{~nm}$. Analysis of the polymer structures revealed curing depths ranging from $22 \pm 16 \mu \mathrm{m}$ to $166 \pm 11 \mu \mathrm{m}$ depending on the laser power and scanning velocity, thus providing the working parameters for precise scaffold production. The average porosity of produced cubic lattice scaffolds was determined to be $44 \pm 9,5 \%$ by gravimetric analysis. Mechanical testing of the cubic lattice demonstrated an uptake of compression stress of up to $1,01 \pm 0,21 \mathrm{MPa}$ at a maximum compression of $74,5 \pm 6,62 \%$.

\section{Conclusion}

In this work we present a methodology to produce load-bearing 3D polymer scaffolds from a biocompatible, photoinitiator-free thiol-ene material. These scaffolds structures can be efficiently produced even on larger scales and their architecture can be specifically designed by CAD in future work. This allows the access to more complex structures and facilitates the generation of artificial, biomechanically challenging tissues and cell environments. 


\section{Rapid Prototyping of Molds for PDMS-based microfluidic chips}

Leona M. Schmidt-Speicher, Karlsruhe Institute of Technology (KIT), Institute of Microstructure Technology (IMT), Karlsruhe, Germany, leona.schmidt-speicher@kit.edu

Tobias Mellert, KIT, IMT, Karlsruhe, Germany, tobias.mellert@gmx.de

Andrea Hurtado, KIT, IMT, Karlsruhe, Germany, andrea.hurtado@kit.edu

Dr. Kerstin Länge, KIT, IMT, Karlsruhe, Germany, kerstin.laenge@kit.edu

Dr. Ralf Ahrens, KIT, IMT, Karlsruhe, Germany, ralf.ahrens@kit.edu

Prof. Dr. Andreas E. Guber, KIT, IMT, Karlsruhe, Germany, andreas.guber@kit.edu

\section{Introduction}

Making microfluidic-chip-prototypes using polydimethylsiloxane (PDMS) to mold the target microstructure is a standard procedure in microfluidic research. Unfortunately, manufacturing these molds by micromilling is a timeconsuming process. In addition, certain structures cannot be milled directly.

We tested, if additive manufacturing might shorten the production of PDMS-molds and enable the direct production of certain structures, that could not be generated directly before. Therefore, we tested the additive manufacturing possibilities available at the IMT in order to estimate if they would be suitable for a rapid PDMS-mold generation.

\section{Methods}

Identical mold structures were manufactured by rapid prototyping using fused filament fabrication (FFF, printers: Ultimaker 3 and Prusa i3 Mk3S), stereolithography (SL, printers: Prusa SL1, MiiCraft+ and Creality LD 002 H) and by micromilling as a reference. The resulting prints were evaluated regarding their moldability and production time. The molded PDMS-microstructures were assessed according to their shape accuracy and transparency.

\section{Results}

The molds could be generated faster by the additive manufacturing processes. Structures $\geq 250 \mu \mathrm{m}$ could be produced with adequate surface quality and moldability. However, structures in these dimensions showed best shape accuracy for squared structures. Structures $\leq 250 \mu \mathrm{m}$ lacked the necessary shape accuracy.

In addition, the drawback that some microstructures could only be milled in their final way and not as negative-moldstructures could be overcome due to a double copying process: the PDMS-cast copied directly from the milled structure was covered with a silane-coat and could then be used as the final mold for molding the target structures in PDMS.

\section{Conclusion}

Thus, the tested additive manufacturing processes clearly showed an advantage regarding the production time and surface quality compared to the micro milling process. However, for smaller structure sizes and/or not squared shaped structures the shape accuracy was not sufficient with the available printers. 


\section{Generalised Image Processing Method for Quantitative Analysis of Nucleus, Cell and Focal Adhesion Clusters in Adherent Cells}

Rajasree Padmakumari Hemachandran Nair, Reutlingen Research Institute, Reutlingen University, Reutlingen, Germany, rajasree.padmakumari_hemachandran_nair@reutlingen-university.de

Rohit Menon, Neura Robotics, Reutlingen, Germany, rohitm1986@gmail.com

Ralf Kemkemer, Applied Chemistry, Reutlingen University, Germany, ralf.kemkemer@reutlingen-university.de

\section{Introduction}

Focal adhesion clusters (FACs) are dynamic and complex structures that help cells to sense physicochemical properties of their environment. Research in biomaterials, cell adhesion or cell migration often involves the visualization of FACs by fluorescence staining and microscopy, which necessitates quantitative analysis of FACs and other cell features in microscopy images using image processing. We developed a simple and robust method and compared it to another method by analysing images from an exemplary biomaterial study with different surface coatings.

\section{Methods}

The effect of different polyelectrolyte multilayer coatings of cell culture substrates on cell adhesion of Human Umblical Vein Enothelial cells (HUVEC) was studied. Fluorescence microscopy images of cells obtained at 65x magnification were quantitatively analysed using ImageJ software. A generalised algorithm for selective segmentation and morphological analysis of FACs, nucleus and cell morphology is implemented. Further, a method for discrimination of FACs near the nucleus and around the periphery is implemented using masks.

\section{Results}

Our method shows uniform sensitivity to colors across the spectrum, thereby enabling to detect differently stained regions of interest (ROI), with optional user adjustable size and color thresholds. An additional selective segmentation using masking enables to discriminate between ROI's located at different positions in the cell, which other methods do not. Our method is additionally robust to fluctuations in image contrast and brightness.

\section{Conclusion}

Our algorithm is able to effectively quantify different morphological characteristics of cell components and shows a high sensitivity and specificity while providing a modular software implementation. 


\section{Development and validation of an experimental setup to investigate auditory neuron survival and outgrowth under clinically relevant elec- trical stimulation using a cochlear implant in an artificial cochlear model}

Tchadarou Abdoul, Department of Sensors and Measurement Technology, Leibniz University Hannover, Hanover, Germany, tchadarouabdoul@googlemail.com

Jana Schwieger, Department of Otolaryngology Hannover Medical School, Hanover, Germany, Schwieger.Jana@mhhannover.de

Thomas Lenarz, Department of Otolaryngology Hannover Medical School, Hanover, Germany, lenarz.thomas@mhhannover.de

Waldo Nogueira, Department of Otolaryngology Hannover Medical School, Hanover, Germany, NogueiraVazquez.Waldo@mh-hannover.de

Verena Scheper, Department of Otolaryngology Hannover Medical School, Hanover, Germany, Scheper.Verena@mhhannover.de

\section{Introduction}

Cochlear implants (CI) can restore hearing to people suffering from sensorineural hearing loss. The CI uses an array of electrodes inserted in the scala tympani to stimulate the spiral ganglion neurons (SGN), which are located in the bony axis of the inner ear. However, the spatial distance between the electrodes and the SGN results in spread of excitation and unfocused stimulation. The distance could be bypassed by a neurite outgrowth towards the CI. It has been shown that electrical stimulation has a positive effect on the neuronal survival and neurite outgrowth. In order to analyse the effect of electrical stimulation on neurite outgrowth and establish a culture system for the development of novel strategies for CI optimization a neurite outgrowth chamber (NOC) allowing clinically relevant stimulation paradigms was developed.

\section{Methods}

Using fused deposition modelling a simplified model of the inner ear consisting of a scala tympani compartment and a neighbouring region serving as model for the Rosenthals' canal was 3D-printed out of biocompatible UV-curing silicone. The model was used as NOC and equipped with a slot to insert the electrode array into the scala tympani compartment whereas SGN isolated from postnatal rats were cultured in the canal region. Sound delivered through loudspeakers playing a radio program was captured by the CI sound processor, positioned outside the NOC, for 17 hours daily during four days. The stimulation paradigms were based on the mean of $\mathrm{N}=25$ patients. Stimulated and unstimulated cells (NC) were stained and microscopically analysed for explant area as well as neurite number and length.

\section{Results}

The NOCs were tightly sealed and the electrode array could be positioned in the scala tympani compartment. The experimental set up allowed cell cultivation and the stimulation resulted in a significantly increased neurite length of around $36 \%$ while explant area and neurite number did not differ to the NC.

\section{Conclusion}

A new in-vitro testing system which considers the anatomy of the cochlear and clinic stimulation conditions has been developed and validated. The validation experiments resulted in increased neurite length. This indicates a progress in bridging the anatomical gap between electrode and stimulated neurons. The next steps consist of implementing outgrowth matrixes and supporting drug delivery to guide the neurites towards the electrode for a more focused and efficient stimulation. 


\section{Low-cost photolithography system for cell biology labs}

Benjamin K. Naggay, Department of Applied Chemistry at Reutlingen University, Reutlingen, Germany Benjamin.Naggay@Reutlingen-University.de

Kerstin Frey, Department of Applied Chemistry at Reutlingen University, Reutlingen, Germany Kerstin.Frey@Reutlingen-University.de

Markus Schneider, Department of Applied Chemistry at Reutlingen University, Reutlingen, Germany

Kiriaki Athanasopulu, Department of Applied Chemistry at Reutlingen University, Reutlingen, Germany

Kiriaki.Athanasopulu@Reutlingen-University.de

Günter Lorenz, Department of Applied Chemistry at Reutlingen University, Reutlingen, Germany Guenter.Lorenz@Reutlingen-University.de

Ralf Kemkemer, Department of Cellular Biophysics at Max Planck Institute for Medical Research, Stuttgart, Germany Department of Applied Chemistry at Reutlingen University, Reutlingen, Germany Ralf.Kemkemer@reutlingen-univer$\underline{\text { sity.de }}$

Soft lithography, a tool widely applied in biology and life sciences with numerous applications, uses the soft molding of photolithography-generated master structures by a polymer. In a first step, master molds are fabricated by photolithography, then $\mu \mathrm{m}$-sized surface features can be transferred to the soft polymer replicas. The replicas can, among others, be used for micro-contact printing $(\mu \mathrm{CP})$ or as substrates with ridges, grooves, columns, or cavities to manipulate or control cell adhesion, proliferation, and migration or even to measure cell-generated forces. In addition, prototypes of microfluidic devices are often produced by soft lithography.

The manufacturing of the master structures by the photolithographic process is the main challenge for many biology labs. It affords costly equipment like a UV mask-aligner and a cleanroom environment is often necessary to avoid dust particles on the samples.

Here we present a low-cost alternative to commercially available UV mask-aligners that can easily be established in biological laboratories. The self-built photolithography system is based on consumer light-emitting UV diodes (LEDs) with simple control electronics and a distant-adjustable sample holder. There is no additional optics required and standard chrome masks as well as printed transparency masks can be used to manufacture master structures with various photoresists such as SU-8 and ma-P1275 HV, or any other resist sensitive in the conventional UV-light (350-400 nm) range. We tested the setup by producing circular, squared or, rectangular surface structures of heights from $10 \mu \mathrm{m}$ to $50 \mu \mathrm{m}$ and diameters ranging from $50 \mu \mathrm{m}$ to $200 \mu \mathrm{m}$ and investigating their quality by SEM.

To achieve a cleanroom-like environment, we built a small laminar-flow chamber based on commercially available HEPA filters enabling a nearly dust-free working space. In combination with a simple spin-coater and heating plate, our system offers a cheap and simple alternative to standard photolithography equipment and allows the reliable production of surface features in the range down to several micrometers. 